\title{
BUCHBESPRECHUNGEN / BOOK REVIEWS
}

Jan Wouters / Tanguy de Wilde / Pierre Defraigne / Jean-Christophe Defraigne (eds.):

China, the European Union and Global Governance, Cheltenham UK, Northampton MA, USA, Edward Elgar 2012, 384 S., 90,00 GBP, Hardcover, ISBN 978-1-78100-426-5

Der vorliegende Sammelband enthält die Beiträge zu einer internationalen Konferenz mit dem Titel "China, the EU and the Restructuring of Global Governance", die am 6./7. Mai 2010 in Brüssel stattfand. Die insgesamt 22 Beiträge wurden von 20 Autoren und den Herausgebern Jan Wouters (Universität Leuven), Tanguy de Wilde d'Estemael (Université catholique de Louvain), Pierre Defraigne (Madariaga-College of Europe Foundation) sowie Jean-Christophe Defraigne (Université Saint- Louis, Brüssel) verfasst. Sie repräsentieren ein breites Spektrum an Expertise und Professionen mit einem deutlichen Schwerpunkt auf Universitäten und EU-Institutionen. Das Buch ist in fünf Abschnitte (,Shifts in the Global Order", „The World Trading System”, "The World Financial and Monetary System", "Climate Change and Energy" sowie "Security and Politics") eingeteilt, die von einer Einleitung der Herausgeber sowie einem Schluss und Ausblick von Wouters und Matthieu Burnay eingerahmt werden.

Die Herausgeber verfolgen mit diesem Konferenzband das doppelte Ziel, einerseits verschiedene Felder von global governance zu betrachten, auf die sich der Aufstieg Chinas auswirkt und andererseits die Reaktionen der EU darauf zu analysieren. Der Hauptgegensatz zwischen China und der EU sei dabei, dass die VR China ein multipolares System anstrebe, während die EU ein System aus universellen Rechten bevorzuge - zwei schwer miteinander zu vereinbarende Visionen.

Der erste Abschnitt des Buches beleuchtet die Verschiebung der globalen Ordnung durch den Aufstieg Chinas in drei Beiträgen: Jean-Christophe Defraigne untersucht die Frage, wie sich der Aufstieg Chinas auf das internationale Machtverhältnis auswirken wird. Für ihn ist Weltpolitik ein Nullsummenspiel, in dem die EU Gefahr läuft, ökonomisch und politisch durch die starken Akteure USA und VR China marginalisiert zu werden. Wang Yiwei legt überzeugend dar, dass China in verschiedenen Dimensionen (als Nation, als regionaler sowie als weltpolitischer Akteur) handelt, die es mit seinen sehr unterschiedlichen Identitäten (sozialistischer Staat, östliche Zivilisation, Entwicklungsland sowie aufsteigende Macht) in Einklang bringen muss. Anhand des Beispiels Klimapolitik kann er nachvollziehbar zeigen, dass Konflikte zwischen China und der EU - zumindest für eine bestimmte Zeitperiode - unvermeidbar sind. Denn China hat neben den weltpolitischen Aspekten auch noch seine eigene innenpolitische und regionale Entwicklung im Blick zu behalten, die zum Teil im Widerspruch mit den Klimaschutzzielen stehen. Stewart Fleming bezweifelt im letzten Beitrag dieses Abschnitts eine dauerhaft einflussreiche Rolle der G20 als ein neues zentrales Forum zur Diskussion internationaler ökonomischer Politik. Für die EU als Mitglied der G20 mahnt er größere Einigkeit und eine stärkere Position an, mit der 
die globalen Interessen der EU diplomatisch und auch militärisch durchgesetzt werden könnten.

Im zweiten Teil des Buches beschäftigen sich drei Beiträge mit dem Welthandelssystem. Wouters und Burnay beschäftigen sich mit der Beziehung zwischen der EU und der VR China innerhalb der Welthandelsorganisation (WTO). Sie meinen, dass die beiden Akteure eine wesentliche Rolle in der WTO spielen, sowohl aufgrund ihrer führenden Positionen in der Weltwirtschaft als auch aufgrund der außerordentlichen Spannungen zwischen ihnen. Die Tatsache, dass sie zunehmend die Mechanismen der WTO nutzen, um ihre Konflikte zu lösen, stärkt zwar einerseits diese multilaterale Institution. Andererseits, so meinen die Autoren, stehe vor allem Chinas instrumentelles Verständnis von Multilateralismus einer dauerhaften Stärkung der WTO noch im Wege. Auch Professor Wang Xiaodong von der Shanghaier Tongji-Universität ist von der entscheidenden Rolle der EU und der VR China für das langfristige Gelingen des WTO-Systems überzeugt. Aus seiner Sicht sind vor allem der Mangel an gemeinsamen Zielen und koordinierten Initiativen, die Verbreitung von bilateralen Freihandelsabkommen sowie das ,single undertaking“, also das Prinzip, dass sich die Verhandler nicht für einzelne Punkte, sondern nur für das „Gesamtpaket“ entscheiden können, für das bisherige Scheitern der Doha Development Agenda-Verhandlungen ursächlich. Professor Wu Chien-Wei von der National Chung Cheng University (Taiwan) vergleicht in seinem Beitrag die Afrikapolitik der EU und Chinas, die sich vor allem durch die entwicklungspolitischen Prinzipien der europäischen Konditionalität und der chinesischen Nichteinmischung in interne Angelegenheiten unterscheiden. $W u$ stellt fest, dass sowohl die EU als auch China in der Praxis schon von ihren Prinzipien abgewichen seien und argumentiert, dass ein trilateraler Dialog zwischen der EU, der VR China und den afrikanischen Staaten unter besonderer Berücksichtigung der afrikanischen Entwicklungsbedürfnisse einen Ausgleich zwischen den beiden Extrempositionen schaffen und damit sinnvolle Strategien für Afrika herbeiführen könne.

Dem dritten Abschnitt über das Weltfinanz- und Währungssystem sind in dem vorliegenden Band vier Aufsätze gewidmet. Pierre Defraigne sieht spätestens seit der Weltfinanz- und -Schuldenkrise dringenden Reformbedarf im Weltfinanz- und Währungssystem, wobei er Reformbedarf insbesondere bei den USA (Sparrate erhöhen), der VR China (Reform des Finanzsektors) sowie der EU (Transformation der Währungsunion in eine politische Union) anmahnt. Michel Aglietta stellt anschließend Überlegungen über eine Reform des Internationalen Währungsfonds (IMF) an. Sie müsste die aufstrebenden Schwellenländer stärker berücksichtigen - nicht zuletzt durch reformierte, den tatsächlichen weltwirtschaftlichen Gegebenheiten angemessenere Abstimmungsmodi und die Abschaffung der ungeschriebenen Vorgabe der europäischen Herkunft des IMF-Exekutivdirektors und für den Fonds insgesamt stärkeres politisches Gewicht mit sich bringen. Silvain Plasschaert geht in seinem Artikel der Frage nach, ob die Chinesische Währung (CNY) tatsächlich unterbewertet ist, wie vor allem die USA seit langem behaupten. In seiner Analyse stellt er klar, dass bestimmte Prämissen der Aufwertungsforderung nicht zutreffen: So sei weder die Bewertung der chinesischen Währung die Ursache für das Handelsbilanzde- 
fizit der USA (und damit für den Verlust von amerikanischen Arbeitsplätzen) noch Chinas Handelsbilanzüberschuss die Ursache für seine enormen Devisenvorräte. Eine Währungsaufwertung hätte zudem so schwerwiegende Folgen für die VR China - Exporte in den wichtigsten Absatzmarkt USA würden sich reduzieren, das Wachstum sich insgesamt verlangsamen -, dass sie sie wohl kaum in Kauf nehmen würde. $Q u$ Bo, Professor an der China Foreign Affairs University in Beijing, zeigt ebenfalls recht einprägsam am Beispiel des internationalen Währungssystems, dass die chinesische Außenpolitik hinsichtlich internationaler Organisationen vorwiegend von innenpolitischen Vorgaben geprägt ist.

Der vierte Abschnitt zeigt mit seinen sechs Beiträgen unter anderem auch, dass die Themen Klimawandel und Energie in der Forschung bisher mehr Aufmerksamkeit erhalten haben als andere. David Belis und Simon Schunz heben die wichtige Rolle hervor, die China und die EU auch in der Klimapolitik spielen. Viel wird davon abhängen, ob sich die VR China langfristig entscheidet, weiter in Form eines Zweiergipfels mit den USA (,G-2“) die eigenen Interessen gegen den Rest der Weltgemeinschaft durchzusetzen oder sich zusammen mit der EU für eine Variante der Klimapolitik einsetzen wird, die auf allgemeingültigen Regeln beruht. Richard Cooper setzt sich in seinem Beitrag für eine pragmatische Herangehensweise im Klimaschutz ein: Dort wo multilaterale Institutionen aufgrund von zu vielen divergierenden Interessen zu keiner Einigung finden können, sollten auch andere Lösungsoptionen, auf die man sich in kleineren Gremien einigen kann, akzeptiert werden. Chen Jingquan, Zweiter Sekretär der Mission der VR China bei der EU, fasst die Argumente der chinesischen Seite in der Klimadebatte zusammen: Die VR China kann und will ihre Entwicklungsbedürfnisse nicht vernachlässigen; Chinas Anteil an der weltweiten Klimabelastung ist aus historischer Perspektive im Vergleich zu demjenigen der Industrieländer relativ gering; zudem hat die VR China schon beachtliche Fortschritte in der Umweltgesetzgebung zu verzeichnen. Ein Faktor, den Chen und auch andere Autoren in diesem Zusammenhang vernachlässigen, ist das schwerwiegende Problem der mangelhaften Umsetzung der in der Tat sehr fortschrittlichen Gesetze im Bereich Umwelt, die ein wesentliches Hindernis auf dem Weg Chinas zu einem klimafreundlichen Akteur darstellt. Pei-fei Chang, David Belis und Hans Bruyninckx analysieren die bisherigen Erfahrungen mit dem Clean Development Mechanism (CDM) in den Beziehungen zwischen der EU und der VR China und kommen zu dem ernüchternden Ergebnis, dass in Anbetracht der bisherigen Erfahrungen die wirksamste und sparsamste Variante des Klimaschutzes doch der in nationalen Grenzen vorgenommene Klimaschutz ist. Bernard Snoy gibt einen Überblick über die Kooperationen und Dialoge der EU mit der VR China zur Energiepolitik. Bram Bujis und Lucia van Geuns weisen in ihrem abschließenden Beitrag zu diesem Abschnitt unter anderem auf das Konfliktpotential im Bereich erneuerbare Energien und auf einen Trend zu „green protectionism“ hin, der den weltweiten Klimaschutzbestrebungen konträr entgegenstünde.

Vier weitere Beiträge sind im Abschnitt über Sicherheitspolitik zusammengefasst. Die Japanwissenschaftlerin Elena Atanassova-Cornelis untersucht die Sicherheitskonstellatio- 
nen in der Region Ostasien. Sie kommt zu dem Schluss, dass die USA zwar weiterhin der Garant für Sicherheit und Stabilität in Ostasien sind, die Chance der EU jedoch darin besteht, die multilateralen Bestrebungen dort $\mathrm{zu}$ bestärken. Tanguy de Wilde d'Estmael analysiert das Waffenembargo, das die EU gegenüber der VR China in der Folge der Niederschlagung der Demokratiebewegung in Beijing 1989 verhängte. Dass die EU sich bis heute noch nicht auf ein Ende dieser Sanktion einigen konnte, zeigt für ihn die wesentliche Schwäche der EU, nämlich die Uneinigkeit ihrer Mitglieder. Hanne Cuyckens geht in ihrem Beitrag der Frage nach, inwieweit die EU und die VR China gemeinsam die Macht der USA in der Region Ostasien ausbalancieren können. Auch Cuyckens kommt zu dem Schluss, dass eine effektivere und vor allem geeinigte Außenpolitik der EU auch in dieser Frage von Nöten sei. Im letzten Beitrag zu diesem Abschnitt untersucht Jagannath P. Panda Chinas Kooperation mit den anderen BRIC-Staaten und sieht darin eine Strategie, mit der China einerseits durch das Bündnis mit anderen Schwellenländern den Grad der eigenen Entwicklung verbergen und andererseits ein Gegengewicht zur Vormachtstellung der USA in der Weltpolitik herstellen möchte.

Wouters und Burnay fassen schließlich die Ergebnisse zusammen und geben einen Ausblick. Erstens sei in Anbetracht des rasanten Aufstiegs der VR China und der zunehmenden Verschärfung globaler Probleme bei gleichzeitiger Legitimitätskrise der internationalen Organisationen eine Reform des Multilateralismus und seiner Institutionen erforderlich. Zweitens ergeben die verschiedenen Analysen, dass China gegenüber dem Multilateralismus nach wie vor ein vorwiegend instrumentelles Verständnis pflege, was zur Folge hat, dass China einerseits nur dann in den internationalen Organisationen kooperiert, wenn es sich davon einen Vorteil für die eigene Entwicklung verspricht, und andererseits auch daran gehindert wird, eine noch verantwortlichere Führungsrolle im internationalen System zu übernehmen. Über die EU haben die Beiträge drittens gezeigt, dass sie weiterhin an interner Uneinigkeit kranke, und ihre starke Dominanz in internationalen Organisationen zunehmend kritisiert werde. Bezüglich der Beziehungen zwischen der EU und der VR China ziehen die Autoren viertens das Fazit, dass diese immer noch durch gegenseitige Fehlwahrnehmungen, konkurrierende Interessen, Wertkonflikte, aber auch durch die Politik anderer Akteure - vor allem der USA - geprägt sind. Sie ziehen daraus drei Schlüsse: Die EU und China müssen das Wissen übereinander erweitern; der Kooperation fehlt es an einer gemeinsamen strategischen Ausrichtung - zum Beispiel könnte die Verbesserung der global governance ein solches strategisches Ziel für beide sein - und schließlich müssen beide ihre Kooperation in multilateralen Organisationen und im Dialog fortsetzen.

Insgesamt bietet der Sammelband ein gemischtes Bild. Er versammelt Aufsätze von sehr unterschiedlicher Qualität - darunter bieten einige erfrischend neue Einsichten über die EU-China-Beziehungen. Insbesondere Aufsätze, die sich sehr detailliert mit vielfach erwähnten, aber bisher wenig analysierten Themen auseinandersetzen - hier möchte ich Wang Xiaodongs Analyse der chinesischen Außenpolitik am Beispiel der Doha-Verhandlungen, Sylvain Passchaerts Einschätzung der RMB-Währungsfrage, den Beitrag über den CDM-Mechanismus von Chang, Belis und Bruyninckx sowie die Analyse über erneuerbare 
Energie in den EU-China-Beziehungen von Buijs und van Geuns besonders hervorheben. Andere Beiträge bleiben hinter den Erwartungen zurück, so wären im Bereich Sicherheitspolitik ausgewogenere und tiefergehende Analysen vorstellbar. Gerade in diesem Abschnitt manifestieren sich die teilweise sehr dominanten europazentrierten Tendenzen des vorliegenden Bandes. So werden beispielsweise für die verschiedenen Perspektiven auf die Region ausschließlich europäische Analysten zitiert, obwohl die Sicht der Länder selbst (v.a. der VR China und der USA) aufschlussreicher gewesen wäre und möglicherweise ein differenzierteres Bild hätten entstehen lassen. Positiv zu bemerken ist, dass mit einigen gängigen Klischees aufgeräumt wird, z.B. ist die Bezeichnung Chinas als Klimakiller Nr. 1 je nachdem, welcher statistische Ansatz verwendet wird, mehr oder weniger zutreffend. Auch werden manche Motive hinter der chinesischen Außenpolitik aufschlussreich erläutert - z.B. in welchem Dilemma die chinesische Führung bei ihrer Verfolgung der zwei einander widersprechenden Ziele Umweltschutz und Entwicklung steckt.

Die Frage, die der Titel von Konferenz und Buch nahelegt, ob China und die EU zu global governance einen Beitrag leisten (können), wird zwar auf vielerlei Weise in den Beiträgen angeschnitten. Jedoch fehlt eine forschungsleitende Frage und vor allem eine Definition von global governance, die diesbezüglich zu noch mehr originellen Ergebnissen hätte führen können. Ein Aspekt von global governance hätte dadurch mehr Aufmerksamkeit erhalten: das Recht. Welche Rolle spielt das Recht für die politischen Entscheider in der VR China - sowohl in seiner Innen- als auch in der internationalen Politik und in den chinesischen (global) governance-Konzeptionen? Wie wird sich das internationale Recht durch den Aufstieg Chinas weiterentwickeln? Dazu findet sich leider kein Kapitel.

Das Buch richtet sich an Leser aus Politik und Wissenschaft mit einem Interesse an den Beziehungen zwischen der EU und der VR China.

Katja Levy, Berlin 